\title{
Coordinated analyses on space weathering signatures on a Fe-sulfide grain from asteroid Itokawa
}

Laura Chaves ${ }^{1}$, Michelle Thompson ${ }^{2}$ and Shoumya Shuvo ${ }^{3}$

${ }^{1}$ Purdue University, west lafayette, Indiana, United States, ${ }^{2}$ Purdue University - Department of Earth, Atmospheric, and Planetary Sciences, United States, ${ }^{3}$ Purdue University, United States

In 2010, the Japan Aerospace Exploration Agency (JAXA) recovered 1534 regolith particles from the Muses-Sea Regio from the near-Earth S-type asteroid Itokawa. S-type (i.e., stony) asteroids have long been hypothesized to be the source of ordinary chondrite (OC) meteorites; however, the stony asteroids present spectral differences in the visible near-infrared region (VNIR) compared to OC's. The spectral discrepancies include the attenuation of characteristic absorption bands, reddening, and darkening attributed to space weathering. Airless body surfaces are susceptible to solar wind irradiation and micrometeoroid bombardment, which modify the chemistry and microstructure of the minerals on these surfaces in a process called space weathering. Petrological analyses on the Itokawa regolith grains show an LL4-6 composition verifying the link between ordinary chondrites and S-type asteroids [1]. Historically, space weathering studies have focused on understanding how silicate minerals, which are the main constituents of lunar samples and ordinary chondrites respond to space weathering. However, Fesulfide minerals have been identified in the Itokawa particles and are important accessory minerals in carbonaceous chondrites reaching concentrations up to $11 \mathrm{wt} \%$ [2].

Previous transmission electron microscopy analyses (TEM) on Itokawa regolith grains have revealed the presence of complete and partially amorphous rims and vesiculated textures similar to the chemical and microstructural signatures identified on lunar regolith grains [3]. In addition to these lunar-style space weathering characteristics, Itokawa samples also contain sulfide nanophase particles (npFeS), a novel space weathering feature that has not been reported previously [4]. The identification of npFeS is important as iron-bearing nanophase particles (npFe) identified in lunar soils are the main contributor to the altered spectral characteristics of airless bodies. Investigating the production of these npFeS particles on Itokawa is critical for understanding how space weathering operates on more compositionally complex regoliths.

In this study, we analyzed the Itokawa particle RB-CV-0121 allocated by JAXA, which was previously identified to contain Fe-sulfide, olivine, and low-Ca pyroxene phases. The sample preparation was performed by adapting the hybrid ultramicrotomy/focused ion beam (FIB) technique to increase the number of sections available for transmission electron microscopy (TEM) analysis [5]. The 26 micronsized particle was embedded in epoxy and then ultramicrotomed using a Leica EM UC7 to prepare sections with an approximate thickness of $50 \mathrm{~nm}$. After ultramicrotomy, we used a Thermo Scientific Helios G4 UX Dual Beam FIB secondary electron microscope (SEM) to create electron-transparent thin sections. The transmission electron microscopy analyses were performed using an FEI Tecnai G2 20200 $\mathrm{kV}$ equipped with an Oxford X-Max silicon drift energy-dispersive X-ray spectroscopy (EDS) detector, an FEI Talos $200 \mathrm{kV}$ TEM, and a monochromated, aberration-corrected $300 \mathrm{keV}$ Thermo Fisher Themis Z FEG TEM, both equipped with Super-X EDS detectors.

The nanometric Fe-sulfides grains are present both as inclusions and on the edge of the silicate-rich particle. Here we focus on the grains located at the edges of the particle (Fig. 1A) because they might have been exposed to the influence of solar wind and micrometeoroid impacts on the surface of Itokawa. High- 
resolution TEM (HRTEM) imaging of one sulfide (Pyrr-1) indicates that the grain is mostly crystalline, with lattice fringes giving d-spacing values of $0.56 \mathrm{~nm}$ (Fig. 1B), which correspond to pyrrhotite $\left(\mathrm{Fe}_{\mathrm{x}-1} \mathrm{~S}\right.$ with $\mathrm{x}=0$ to 2 ). However, HRTEM imaging reveals the presence of an outer rim with an approximate thickness of $5 \mathrm{~nm}$. We performed EDS line scans from the grain interior to the rim to investigate the sample's compositional changes (Fig. 2B, 2C). EDS data shows sulfur depletion upper $5 \mathrm{~nm}$ of the sulfide grain (Fig. 2A), which corresponds to the outer rim identified in Fig. 1A. We hypothesize that this rim results from sputtering produced by solar wind irradiation as no chemical evidence of vapor deposits was found (i.e., enrichments on $\mathrm{Ca}, \mathrm{Si}$, or $\mathrm{Mg}$ ). To investigate the response of $\mathrm{Fe}$-sulfides to solar wind irradiation, we will use the Stopping and Range of Ions in Matter (SRIM) code to simulate ion-atom collisions.

Previous studies have identified the presence of a disordered rim on a pyrrhotite Itokawa grain [4], and ion irradiation experiments on troilite (FeS) have also produced sulfur depletion [6]. The identification of sulfur depletion on Itokawa grains is important as this element deficiency has also been identified in asteroid Eros' surface by the X-ray spectrometer aboard the Near-Earth Asteroid Rendezvous (NEAR) Shoemaker spacecraft [7]. These findings on Itokawa samples suggest that space weathering might be one of the causes driving sulfur depletion on asteroidal surfaces. The results obtained in this study will be compared to analysis on four other Fe-sulfide bearing Itokawa regolith particles allocated by JAXA and ion and laser irradiation experiments on analog materials.
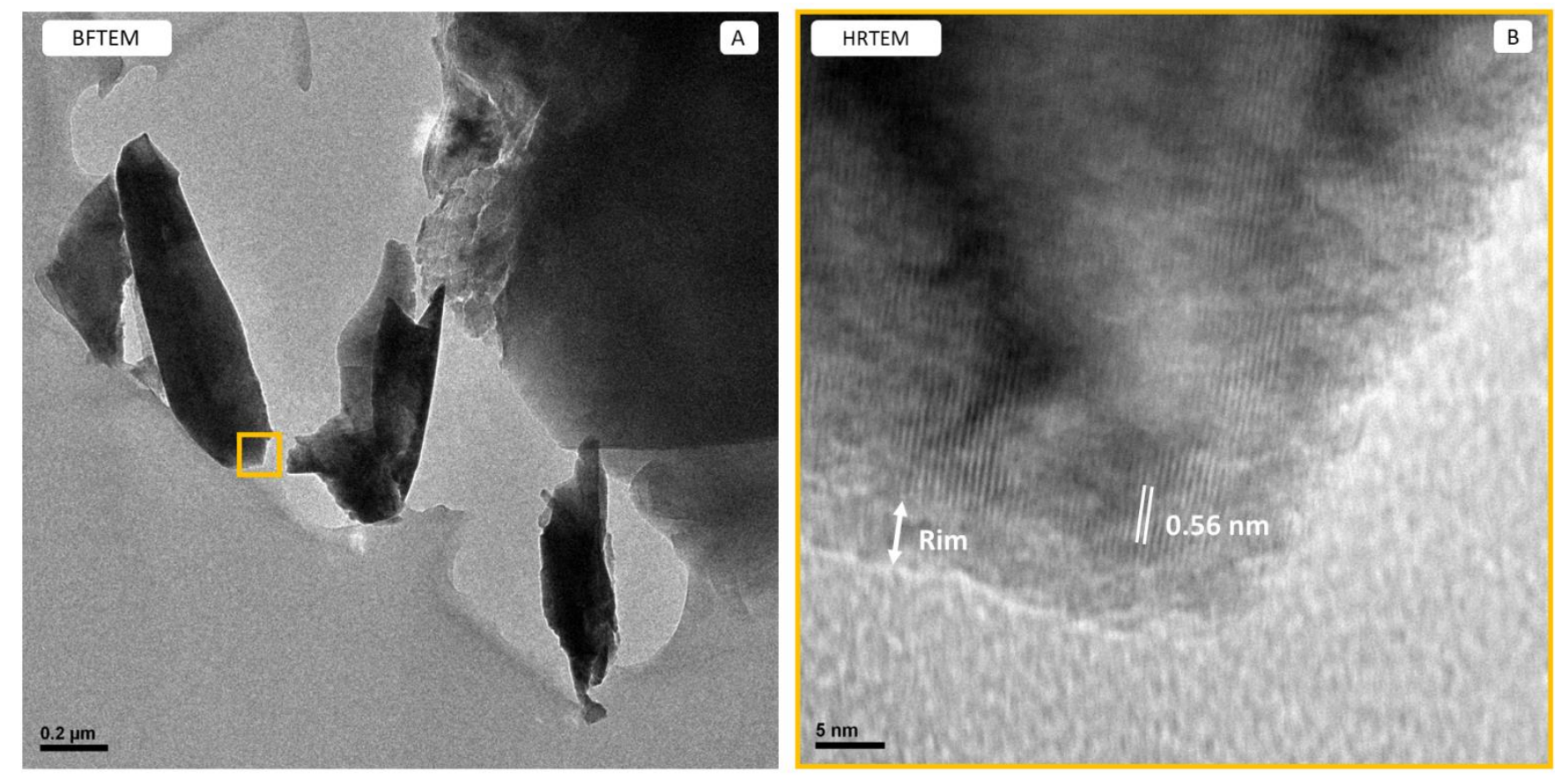

Figure 1. TEM images of Fe-sulfides on the Itokawa particle RB-CV-0121. a) Bright field transmission electron microscope (BFTEM) image showing the location of the sulfides. b) HRTEM image showing the $0.56 \mathrm{~nm}$ wide lattice fringes and the outer rim. 

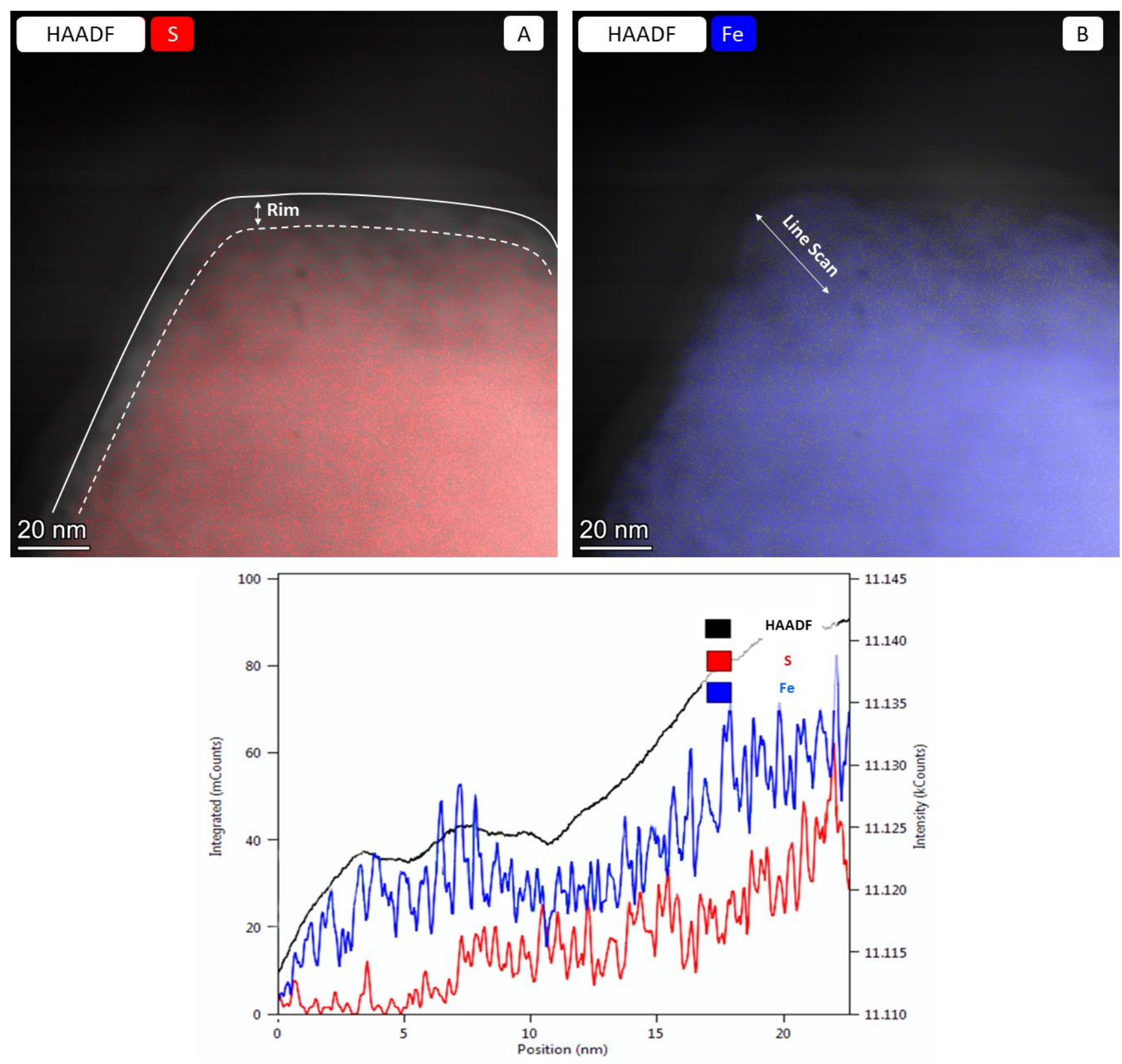

Figure 2. High angle annular dark field (HAADF) images and EDS line scan of Pyrr-1. a) S map showing depletion in the upper $5 \mathrm{~nm}$ rim compared to the b) Fe map. c) EDS line scan of the outer $25 \mathrm{~nm}$ of the grain showing the variation of $\mathrm{Fe}$ and $\mathrm{S}$ along the profile.

\section{References}

[1] Nakamura et al., 2011. Itokawa dust particles: a direct link between S-type asteroids and ordinary chondrites. Science 333, 1113-1116.

[2] Bland P. A. et al., 2004. Modal mineralogy of carbonaceous chondrites by X-ray diffraction and Mössbauer spectroscopy. Meteoritics \& Planetary Science 39, 3-16.

[3] Thompson M. S. et al., 2014. Microchemical and structural evidence for space weathering in soils from asteroid Itokawa. Earth, Planets and Space, 66:89. 
[4] Keller L. P. et al., 2014. A transmission electron microscope study of Itokawa regolith grains. Earth, Planets and Space, 66:71.

[5] Berger E. L. \& Keller L. P., 2015. A hybrid ultramicrotomy-FIB technique for preparing serial electron transparent thin sections from particulate samples. Microscopy Today, 18-22.

[6] Loeffler M. J. et al., 2008. Laboratory simulations of sulfur depletion at Eros. Icarus 195, 662 - 629. [7] Nittler L. R. et al., 2001. X-ray fluorescence measurements of the surface elemental composition of Asteroid 433 Eros. Meteoritics \& Planetary Science 36, 1673-1695. 